\title{
Journal of Materials Chemistry
}

\section{Flying-seed-like liquid crystals $3^{\dagger}$ : New guideline for induction of mesomorphism by using bulky groups instead of long alkyl chains}

\author{
Ayaha Hachisuga ${ }^{a}$, Miho Yoshioka ${ }^{a}$, Kazuchika Ohta*a and Tomoyuki Itaya ${ }^{b}$ \\ Received (in $X X X, X X X) X$ th $X X X X X X X X X 20 X X$, Accepted Xth $X X X X X X X X X 20 X X$ \\ ${ }_{5}$ DOI: 10.1039/b000000x
}

In our previous work, we synthesized a series of phthalocyanine-based flying-seed-like compounds, (nPh-PhO) $)_{\mathbf{4}} \mathbf{P c C u}(\mathrm{n}=3,2,1$ and 0 in $\mathbf{n P h}-\mathbf{P h O}$ : a, b, $\mathbf{c}$ and $\mathbf{d})$, and revealed that each of them shows a columnar liquid crystalline phase $\left(\mathrm{Col}_{\text {ho }}\right.$ or $\left.\mathrm{Col}_{\text {rho }}\right)$ from rt to an extremely high temperature near $500{ }^{\circ} \mathrm{C}$. Thus, we could obtain columnar liquid crystalline phases for discotic compounds by using bulky 10 substituents (a d) instead of long alkyl chains. In order to furthermore study the utility of these bulky substituents (a d) on induction of mesomorphism for the other shaped molecules, we have synthesized novel flying-seed-like calamitc phenoxybenzoic acid derivatives, nPh-PhOBA (3a d), and their Mitsubishi-Mark-shaped terbium complexes, (nPh-PhOBA) ${ }_{3} \mathbf{T b} \cdot \mathbf{m M e O H}(\mathbf{4 a} \sim \mathbf{d})$, in this work. Very interestingly, the mesomorphism of the phenoxybenzoic aids derivatives $\mathbf{n P h}-\mathbf{P h O B A}(\mathrm{n}=0 \sim 3)$ was 15 induced to show a mesophase $\left(M_{x}\right)$ only by the bulky substituent $\mathbf{d}(n=0)$, whereas the mesomorphism of their corresponding terbium complexes (nPh-PhOBA) $)_{3} \mathbf{T b}(\mathbf{I I I})(\mathrm{n}=0 \sim 3)$ was induced to show rectangular columnar $\left(\mathrm{Col}_{r}\right)$ mesophases by the bulky substituents $\mathbf{c}$ and $\mathbf{d}(\mathrm{n}=1,0)$. On the other hand, the mesomorphism of the PcCu complexes (nPh-PhOBA) $)_{4} \mathbf{P c C u}(n=0 \sim 3)$ in our previous work was induced by all the bulky substituents $\mathbf{a}, \mathbf{b}, \mathbf{c}$ and $\mathbf{d}(\mathrm{n}=0 \sim 3)$. Thus, it becomes apparent that both calamitic and 20 columnar mesomorphism can be induced by substitution of bulky groups instead of long alkyl chains, and that balance of core size and bulkiness of substients is very important to obtain mesomorphism in flying-seed-like compounds. We believe that this is a new guideline for induction of mesomorphism by using bulky groups instead of long alkyl chains.

\section{${ }_{25}$ 1. Introduction}

It is well-known that molecular shapes of most liquid crystals are rod-like (calamitic) or disk-like (discotic). Furthermore, both of the molecular structures commonly have a rigid flat core and flexible long chains in the periphery. When such shape of 30 compounds are heated, the flexible long chains melt at first and the rigid flat cores do not melt but still aggregate each other. This pre-melting of peripheral long chains originates the soft part to induce liquid-crystalline phases (mesophases). Hence, it is generally believed that appearance of mesomorphism should 35 require both a rigid flat core and long flexible chains in a molecule. However, in about 101,000 of liquid crystals known until now it has been reported that a very few liquid crystalline compounds are out of this general criterion and they have neither a rigid flat core nor long flexible chains in their molecules ${ }^{1) 9}$.

40 In 1911, Vorländer reported that alkali metal carboxylates, $\mathbf{P h}_{2}$ AcONa, $\left(\mathrm{CH}_{3}\right)_{2} \mathrm{AcOK}$ and $\left(\mathrm{C}_{2} \mathrm{H}_{5}\right)_{2} \mathrm{AcOK}$, shown in Fig.1 exhibit liquid crystalline phases, although they have neither a flat core nor long chains ${ }^{1)}$. However, these interesting liquid crystals have been forgotten for about 100 years. It may be attributable to 45 very high liquid crystalline temperature regions and their molecular structures totally different from general calamitic and discotic liquid crystals.
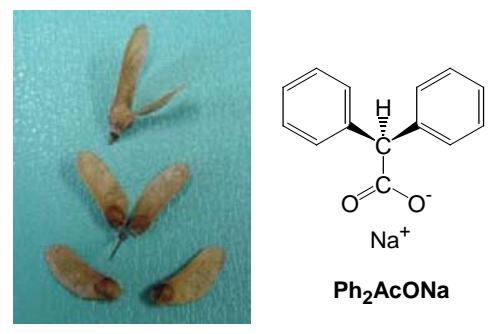<smiles>C[C](C)C(=O)[O-]</smiles><smiles>CCC(CC)C(=O)[O-]</smiles>

$\mathrm{Ph}_{2} \mathrm{AcONa}$

$\left(\mathrm{CH}_{3}\right)_{2} \mathrm{AcOK} \quad\left(\mathrm{C}_{2} \mathrm{H}_{5}\right)_{2} \mathrm{AcOK}$

Fig. 1 Photograph of maple seeds and examples of flying-seed-like liquid 50 crystals: Refs. $1 \sim 4$ and 6.

In 2006 we revealed by using temperature-dependent X-ray diffraction technique that $\mathbf{P h}_{\mathbf{2}} \mathbf{A c O N a}$ shows a hexagonal columnar $\left(\mathrm{Col}_{h}\right)$ mesophase, and $\left(\mathbf{C H}_{3}\right)_{2} \mathbf{A c O K}$ and $\left(\mathbf{C}_{2} \mathbf{H}_{5}\right)_{2}$ AcOK show a calamitic mesophase of smectic A $\left(S_{A}\right)$ ${ }_{55}$ phase $^{6)}$. Nevertheless, it was surprising for us that they have no long chains necessary for formation of soft parts in liquid 
crystalline phase. Therefore, we thought that these bulky substituents of $\mathbf{P h}_{2^{-}},\left(\mathbf{C H}_{3}\right)_{2^{-}}$and $\left(\mathbf{C}_{2} \mathbf{H}_{5}\right)_{2}$-groups might form soft parts by free rotation, like as flying-seeds as shown in the photograph of maple seeds in Fig. 1. Accordingly, we named this 5 type of liquid crystals as "flying-seed-like liquid crystals"6) . They are totally different from conventional calamitic and discotic liquid crystalline compounds.
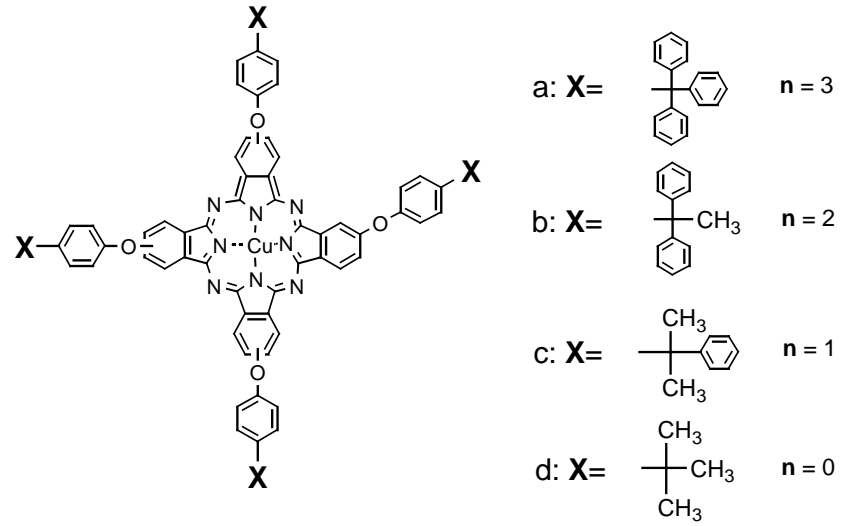

(nPh-PhO) ${ }_{4} \mathrm{PcCu}$

Fig. 2 Molecular formula of phthalocyanine-based flying-seed-like 10

liquid crystals, (nPh-PhO) ${ }_{4} \mathbf{P c C u}$, in our previous work: Refs.10 12.

In 2004 and 2009, Usol'tseva and her co-workers reported that a phthalocyanine (Pc) compound (3Ph-PhO) ${ }_{4} \mathbf{P c C u}$ exhibits a $\mathrm{Col}_{\mathrm{h}}$ mesophase ${ }^{10), 11)}$. Although it has a rigid flat core, it has no long alkyl chains but bulky triphenylmethyl-phenoxy groups (a in ${ }_{15}$ Fig. 2) in the periphery. The present bulky triphenylmethylphenoxy group (a) very resembles $\mathbf{P h}_{2}$-group in a previous flying-seed-like liquid crystal $\mathbf{P h}_{\mathbf{2}} \mathbf{A c O N a}$ mentioned above (Fig. 1). Hence, we considered that this Pc compound (3Ph$\mathbf{P h O})_{4} \mathbf{P c C u}$ was one of the flying-seed-like liquid crystals, and 20 that the bulky triphenylmethylphenoxy groups (a) also formed soft parts in the mesophase. To certify this idea, we have synthesized four phthalocyanine-based flying-seed-like compounds, (nPh-PhO) ${ }_{4} \mathbf{P c C u}(\mathrm{n}=3 \sim 0)$, by using a series of bulky substituents (a d in Fig. 2). Very interestingly, each of 25 them shows a columnar liquid crystalline phase $\left(\mathrm{Col}_{\text {ho }}\right.$ or $\left.\mathrm{Col}_{\text {rho }}\right)$ from rt to an extremely high temperature near $500{ }^{\circ} \mathrm{C}^{12)}$. Thus, it was proved that columnar liquid crystalline phases can be induced for discotic compounds also by using bulky substituents (a d in Fig. 2) instead of long alkyl chains.

30 We wish to furthermore study the utility of these bulky substituents (a d in Fig. 2) on induction of mesomorphism for the other shaped molecules, so that in this work we have synthesized novel flying-seed-like calamitc phenoxybenzoic acid derivatives, nPh-PhOBA (3a $\mathbf{d}$ in Scheme 1), and their ${ }_{35}$ Mitsubish-Mark-shaped $^{13)}$ terbium complexes, (nPhPhOBA) ${ }_{3} \mathbf{T b} \cdot \mathbf{m M e O H}(\mathbf{4 a} \sim \mathbf{d}$ in Scheme 1), to investigate their mesomorphism.

\section{Experimental}

\section{2-1. Synthesis}

40 Scheme 1 shows synthetic route for the present flying-seedlike calamitc nPh-PhOBA (3a d) compounds and MitsubishiMark-shaped (nPh-PhOBA)3Tb $\cdot \mathbf{m M e O H}(\mathbf{4 a} \sim \mathbf{d})$ complexes.
The starting materials, phenol derivatives 2a,c and d, were purchased from Tokyo Chemical Industry, and they were used 45 without further purification. Since the phenol derivative $\mathbf{2} \mathbf{b}$ was not commercially available, it was prepared as shown in this scheme. Commercially available anisole was reacted with 1,1diphenylethanol to obtain 4-[1,1-diphenylethoxy]anisole $\mathbf{1 b}$, and then it was demethylated with hydrobromic acid by using the 50 method of Evangelista et al. ${ }^{14)}$ to afford the phenol derivative $\mathbf{2} \mathbf{b}$. The phenol derivatives $\mathbf{2 a} \sim \mathbf{d}$ were reacted with $p$-nitrobenzoic acid to obtain the corresponding 4-[4-(bulky-groupsubstituted)phenoxy]benzoic acids, nPh-PhOBA (3a d). The phenoxybenzoic acids 3a $\mathbf{d}$ were metalated with terbium(III)

55 nitrate hexahydrate in basic conditon to afford the solvated derivatives (nPh-PhOBA)3Tb・ $\mathbf{m M e O H ~ ( 4 a \sim d ) . ~}$

The detailed procedures are described only for the representative derivatives, $\mathbf{2} \mathbf{b}, \mathbf{3 a}, \mathbf{4 a}$ and $\mathbf{4 c}$. Almost the same procedures for $\mathbf{3 b}, \mathbf{3} \mathbf{c}$ and $\mathbf{3 d}$ are described in the supplementary 60 information file.
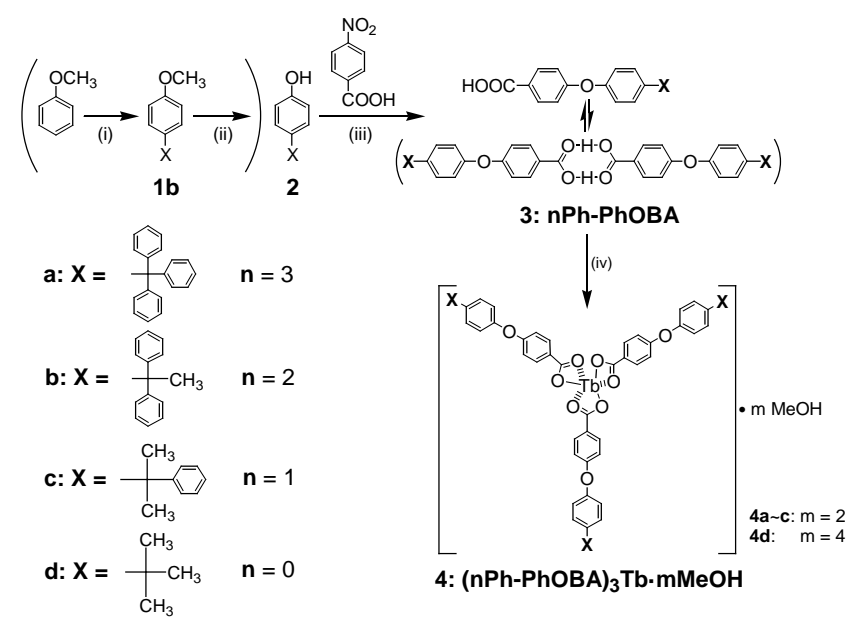

4: (nPh-PhOBA) ${ }_{3} \mathrm{~Tb} \cdot \mathrm{mMeOH}$

Scheme 1 Synthetic route for novel flying-seed-like calamitic nPhPhOBA (3a d) and Mitsubishi-Mark-shaped (nPh-PhOBA) ${ }_{3}$ Tb •

$65 \quad \mathbf{m M e O H}(\mathbf{4 a} \sim \mathbf{d})$ liquid crystals. (i) X-OH, polyphosphoric acid; (ii) 48\% $\mathrm{HBr}$ aq.sol. / $\mathrm{HOAc}$; (iii) (1) $\mathrm{K}_{2} \mathrm{CO}_{3} / \mathrm{DMF} / p$-nitrobenzoic acid (2) $\mathrm{H}^{+}$, (iv) $\left(\mathrm{NO}_{3}\right)_{3} \mathrm{~Tb} \cdot 6 \mathrm{H}_{2} \mathrm{O} / \mathrm{MeOH} /$ ethylene glycol / $\mathrm{NaOH}$ for $\mathbf{4 a}, \mathbf{b}$; $\left(\mathrm{NO}_{3}\right)_{3} \mathrm{~Tb} \cdot 6 \mathrm{H}_{2} \mathrm{O} / \mathrm{MeOH} / \mathrm{NaOH}$ for $\mathbf{4 c , d}$

\section{4-(1,1-diphenylethyl)phenol (2b)}

70 Into a $200 \mathrm{ml}$ of three-neched flask, anisole (1.1g, 10mmol), 1,1diphenylethanol (2.0 g, 10mmol) and polyphosphoric acid (20 g, $200 \mathrm{mmol}$ ) were poured. It was heated with stirring at $100{ }^{\circ} \mathrm{C}$ for 23 hours. After cooling to rt, the reaction mixture was extracted with diethylether and washed with $\mathrm{NaCl}$ saturated aqueous 75 solution. The organic layer was dried over sodium sufate and the evaporated in vacuo. Into a $100 \mathrm{ml}$ of three-neched flask, $2.5 \mathrm{~g}$ of the residue, $58 \mathrm{ml}$ of glacial acetic acid and $14.5 \mathrm{ml}$ of $48 \%$ hydrobromic acid aqueous solution were poured. It was heated with stirring at $100{ }^{\circ} \mathrm{C}$ for 16 hours. After cooling to rt, the 80 reaction mixture was extracted with $\mathrm{CHCl}_{3}$ and washed with $\mathrm{NaCl}$ saturated aqueous solution. The organic layer was dried over soduim sulfate and then dried in vacuo. The residue was purified by column chromatography (120 g of silica gel, $\mathrm{CH}_{2} \mathrm{Cl}_{2}$, $\mathrm{Rf}=0.33$ ) to afford $0.68 \mathrm{~g}$ of ocherous solid was obtained in a ${ }_{85} 25 \%$ yield.

IR(KBr): 3487 (Ar- OH) $\mathrm{cm}^{-1}$ 
${ }^{1} \mathrm{H}-\mathrm{NMR}$ (DMSO-d $\mathrm{d}_{6}$ : TMS) $\delta 2.32\left(3 \mathrm{H}, \mathrm{s},-\mathrm{CH}_{3}\right), 7.02 \sim 7.24(14 \mathrm{H}$, m, aromatic $-H)$, 9.44(1H,s,O-H)

\section{4-(4-tritylphenoxy)benzoic acid (3a)}

A $100 \mathrm{ml}$ of three-necked flask with a condenser was dried by a 5 heat gun under nitrogen atmosphere. Into this flask, $p$ nitrobenzoic acid ( $0.18 \mathrm{~g}, 1.1 \mathrm{mmol})$, freshly distilled dry DMF (5 $\mathrm{ml}), \mathrm{K}_{2} \mathrm{CO}_{3}(0.35 \mathrm{~g}, 2.5 \mathrm{mmol}$ ), and 4-triphenylmethylphenol (2a: $0.44 \mathrm{~g}, 1.3 \mathrm{mmol}$ ) was poured. It was heated with stirring under dry nitrogen atmosphere at $110^{\circ} \mathrm{C}$ for 6 hours. After cooling to rt, 10 it was neutralised by adding $10 \%$ hydrochloric acid dilute aqueous solution, and then extracted with diethyl ether and washed with with $\mathrm{NaCl}$ saturated aqueous solution. The organic layer was dried over sodium sulphate and dried in vacuo. The residue was recrystallised from ethanol to afford $0.33 \mathrm{~g}$ of white 15 solid in a $67 \%$ yield. M.p.: see Table 2.

IR(KBr): 3489 ( - OH), $1710(\mathrm{C}=\mathrm{O}), 1280$ (ether) $\mathrm{cm}^{-1}$

${ }^{1} \mathrm{H}-\mathrm{NMR}$ (DMSO-d $\mathrm{d}_{6}$ : TMS) $\delta 6.66(2 \mathrm{H}, \mathrm{d}, \mathrm{J}=9.1 \mathrm{~Hz}$, aromatic $-H), 6.93(2 \mathrm{H}, \mathrm{d}, \mathrm{J}=9.0 \mathrm{~Hz}$, aromatic $-H), 7.08 \sim 7.32(19 \mathrm{H}, \mathrm{m}$, aromatic $-H)$, $9.36(1 \mathrm{H}, \mathrm{s}, \mathrm{COO}-H)$

20 (3Ph-PhOBA) ${ }_{3} \mathbf{T b} \cdot 2 \mathrm{MeOH}(4 \mathrm{a})$

Into a 50ml Erlenmeyer, $\mathrm{NaOH}(0.048 \mathrm{~g}, 1.2 \mathrm{mmol})$, terbium(III) nitrate hexahydrate $(0.14 \mathrm{~g}, 0.30 \mathrm{mmol}), 4$-(4-tritylphenoxy) benzoic acid (3a: $0.46 \mathrm{~g}, 1.0 \mathrm{mmol}), \mathrm{MeOH}(5 \mathrm{ml})$ and ethylene glycol $(0.5 \mathrm{ml})$ were added, and it was stirred at rt for 24 hours.

25 The resulted precipitate was collected by filtration and washed with $\mathrm{MeOH}$. The residue was dried in vacuo to afford 0.31g of white crystal in a $60 \%$ yield.

\section{(2Ph-PhOBA) ${ }_{3}$ Tb・2MeOH (4b)}

As the same manner as $4 \mathbf{a}$, it could be prepared to afford $0.30 \mathrm{~g}$ of 30 white crystal in a $65 \%$ yield.

\section{(1Ph-PhOBA) $)_{3} \mathrm{~Tb} \cdot 2 \mathrm{MeOH}(4 \mathrm{c})$}

Into a 50ml Erlenmeyer, $\mathrm{NaOH}(0.048 \mathrm{~g}, 1.2 \mathrm{mmol})$, terbium(III) nitrate hexahydrate $(0.14 \mathrm{~g}, \quad 0.30 \mathrm{mmol}), \quad 4$-[4-(1-methyl-1phenylethyl) phenoxy]benzoic acid (3c: $0.33 \mathrm{~g}, 1.0 \mathrm{mmol}$ ) and ${ }_{35} \mathrm{MeOH}$ ( $5 \mathrm{ml}$ ) were added, and it was stirred at rt for 24 hours. The resulted precipitate was collected by filtration and washed with $\mathrm{MeOH}$. The residue was dried in vacuo to afford $0.25 \mathrm{~g}$ of white crystal in a $64 \%$ yield.

\section{(OPh-PhOBA) ${ }_{3} \mathrm{~Tb} \cdot 4 \mathrm{MeOH}(4 \mathrm{~d})$}

${ }_{40}$ As the same manner as $4 \mathbf{c}$, it could be prepared to afford $0.20 \mathrm{~g}$ of white crystal in a $55 \%$ yield.

Table 1 Elemental analysis data of (nPh-PhOBA) ${ }_{3} \mathbf{T b} \cdot \mathbf{m M e O H}, 4 \mathbf{4} \sim \mathbf{d}$

\begin{tabular}{|c|c|c|c|c|}
\hline \multirow{2}{*}{ Compound } & \multirow{2}{*}{ lecular formula } & \multicolumn{3}{|c|}{ Elemental analysis: Found (Calcd)/ \% } \\
\hline & & $\mathrm{C}$ & $\mathrm{H}$ & $\mathrm{N}$ \\
\hline 4a: (3Ph-PhOBA) ${ }_{3} \mathrm{~Tb} \cdot 2 \mathrm{MeOH}$ & $\mathrm{C}_{98} \mathrm{H}_{77} \mathrm{O}_{11} \mathrm{~Tb}$ & $73.83(74.05)$ & $4.85(4.88)$ & $0.02(0.00)$ \\
\hline $4 \mathrm{~b}:(2 \mathrm{Ph}-\mathrm{PhOBA})_{3} \mathrm{~Tb} \cdot 2 \mathrm{MeOH}$ & $\mathrm{C}_{83} \mathrm{H}_{71} \mathrm{O}_{11} \mathrm{~Tb}$ & $70.94(71.04)$ & $5.02(5.10)$ & $0.01(0.00)$ \\
\hline 4c: (1Ph-PhOBA) ${ }_{3} \mathrm{~Tb} \cdot 2 \mathrm{MeOH}$ & $\mathrm{C}_{68} \mathrm{H}_{65} \mathrm{O}_{9} \mathrm{~Tb}$ & $66.85(67.10)$ & $5.21(5.38)$ & $0.10(0.00)$ \\
\hline 4d: (OPh-PhOBA) ${ }_{3} \mathrm{~Tb} \cdot 4 \mathrm{MeOH}$ & $\mathrm{C}_{55} \mathrm{H}_{67} \mathrm{O}_{13} \mathrm{~Tb}$ & $62.07(62.14)$ & $6.43(6.35)$ & $0.01(0.00)$ \\
\hline
\end{tabular}

\section{2-2. Measurements}

The compounds synthesized here were identified by using FT-IR 45 (Nicolet NEXUS670 FT-IR), ${ }^{1}$ H-NMR (BRUKER DRX-400), elemental analysis (Perkin-Elmer elemental analyzer 2400), and thermogravity analysis (Rigaku Thermo plus TG8120). Phase transition behaviour of the present compounds (3a $\sim \mathbf{d}$ and $4 \mathbf{a} \sim \mathbf{d}$ ) was observed with polarizing optical microscope (Nikon ${ }_{50}$ ECLIPSE E600 POL) equipped with a Mettler FP82HT hot stage and a Mettler FP-90 Central Processor, and a Shimadzu DSC-50 differential scanning calorimeter. For the mesophase identification, we employed a new small angle X-ray diffractometer (Bruker Mac SAXS System) equipped with a 55 temperature-variable sample holder adopted a Mettler FP82HT hot stage. Figs. S1 and S2 illustrate the setup of the SAXS system and the setup of the temperature-variable sample holder, respectively. As can be seen from Fig. S1, the generated X-ray is bent by two convergence monochrometers to produce point $\mathrm{X}$-ray ${ }_{60}$ beam (diameter $=1.0 \mathrm{~mm}$ ). The point beam runs through holes of the temperature-variable sample holder. As illustrated in Fig. S2, into the temperature-variable sample holder of Mettler FP82HT hot stage, a glass plate $(76 \mathrm{~mm} \times 19 \mathrm{~mm} \times 1.0 \mathrm{~mm})$ having a hole (diameter $=1.5 \mathrm{~mm}$ ) is inserted. The hole can be charged with a ${ }_{65}$ powder sample (ca. $1 \mathrm{mg}$ ). The measurable range is from $3.0 \AA$ to $100 \AA$ and the temperature range is from rt to $375^{\circ} \mathrm{C}$. This SAXS system enabled us to greatly reduce the amount of samples. The measurement time only needed 60 300 seconds, which was efficient to suppress the sample decomposition. Temperature70 dependent fluorescence spectra of $\mathbf{4} \mathbf{c}, \mathbf{d}$ were recorded by using a Horiba Fluorolog FL3-21-NIR-OS spectrofluorometer equipped with a handmade hot plate ${ }^{15)}$ available from rt to $400^{\circ} \mathrm{C}$ with a temperature controller.

\section{Results and Discussion}

\section{3-1. Synthesis}

As can be seen from Scheme 1, the fourth synthetic step (iv) from the phenoxybenzoic acid derivatives to the terbium complexes was successful for $\mathbf{4} \mathbf{c}, \mathbf{d}$ by using a single reaction solvent of $\mathrm{MeOH}$. On the other hand, it was not successful for $\mathbf{4 a}, \mathbf{b}$ by using 80 the single solvent of $\mathrm{MeOH}$. It was attributable to the low solubilities of $\mathbf{3 a}, \mathbf{b}$ in pure $\mathrm{MeOH}$ which were resulted from the big number of phenyl groups in the bulky substituents. When a mixture solvent of $\mathrm{MeOH}$ and ethylene glycol $(10: 1 \mathrm{v} / \mathrm{v})$ was adopted for $\mathbf{3 a}, \mathbf{b}$, the metallation successfully took place to afford 85 the terbium complexes $\mathbf{4 a}, \mathbf{b}$.
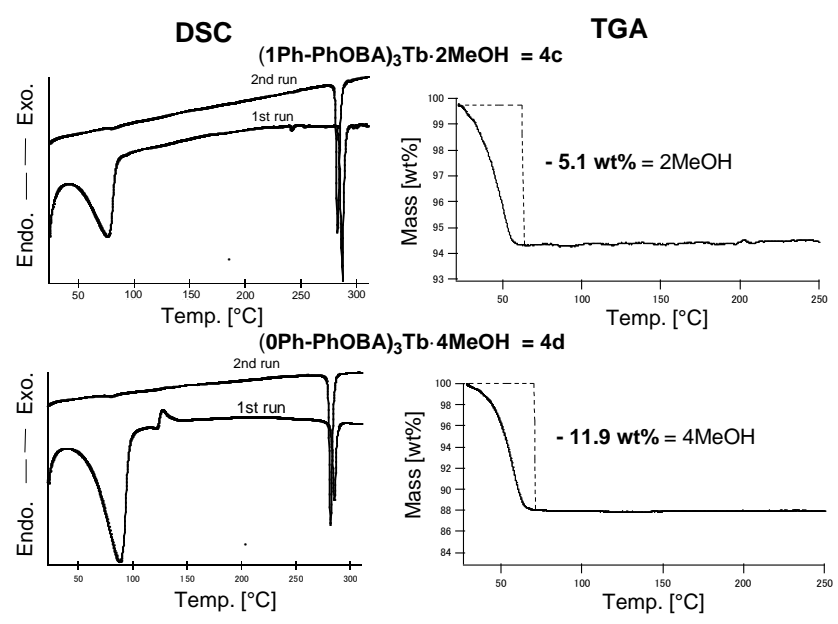

Fig. 3 DSC and TGA thermograms of (1Ph-PhOBA $)_{3} \mathbf{T b} \cdot \mathbf{2 M e O H}(4 \mathrm{c})$ and (0Ph-PhOBA) $)_{3} \mathrm{~Tb} \cdot 4 \mathrm{MeOH}(4 \mathrm{~d})$

90 As can be seen from the elemental analysis data in Table 1, each of the terbium complexes $4 \mathbf{4} \sim \mathbf{c}$ contained two $\mathrm{MeOH}$ 
molecules as the crystal solvent in the freshly prepared (virgin) sample. On the other hand, the terbium complex 4d contained four $\mathrm{MeOH}$ molecules as the crystal solvent. The number of $\mathrm{MeOH}$ molecules ( $\mathrm{m}$ in Scheme 1) could be also confirmed from 5 the thermogravity analyses (TGA) as shown in Fig. 3. As can be seen from the DSC thermograms in this figure, each of the virgin samples (the first heating runs) of $\mathbf{4 c}$,d gave a large endothermic peak with an onset at $c a .50{ }^{\circ} \mathrm{C}$, whereas it disappeared in the second heating runs. As can be seen from the TGA thermograms 10 in this figure, the virgin samples of $\mathbf{4 c}$ and $\mathbf{4 d}$ gave $5.1 \%$ and $11.9 \%$ of weight losses at $\mathrm{ca} .50{ }^{\circ} \mathrm{C}$, respectively. The weight losses of $\mathbf{4 c}$ and $\mathbf{4 d}$ exactly correspond to the weights of two and four $\mathrm{MeOH}$ molecules, respectively. It is consistent with the elemental analysis data in Table 1 mentioned above. The terbium 15 complex 4d only contains four $\mathrm{MeOH}$ molecules. It may be attributed to more space around the Mitsubishi-Mark-shaped terbium complex $\mathbf{4 d}(\mathrm{n}=0)$ in comparison with the other bulky pheny-group-substituted terbium complexes $4 \mathbf{a} \sim \mathbf{c}(n=3 \sim 1)$.

\section{3-2. Mesomorphic properties}

20 The phase transition sequences for the flying-seed-like calamitic phenoxybenzoic acid derivatives nPh-PhOBA (3a $\sim \mathbf{d}$ ) and their corresponding desolventized Mitsubishi-Mark-shaped terbium complexes (nPh-PhOBA) $)_{3} \mathbf{T b}$ (3a $\sim$ d) are summarized in Table 2.

\section{${ }_{25}$ Mesomorphism of 0Ph-PhOBA (3d)}

It is well known and established that long alkyl-chain-substituted benzoic acid derivatives form dimers by hydrogen bonding and show mesomorphism ${ }^{16-18)}$. The present phenoxybenzoic acid derivatives nPh-PhOBA (3a d) may also form dimers as 30 illustrated in Scheme 1.

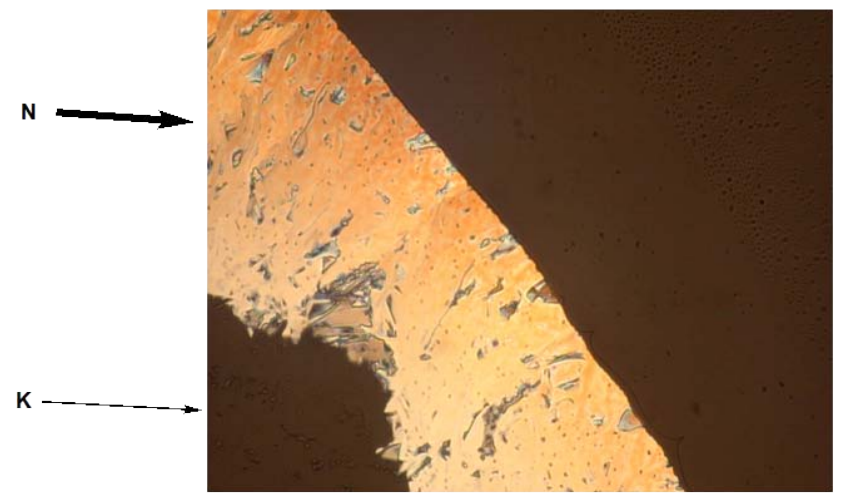

Fig. 4 Photomicrograph of $\mathbf{0 P h}-\mathbf{P h O B A}(\mathbf{3 d})$ at $22{ }^{\circ} \mathrm{C}$. The crystalline phase $K$ was changing into an unidentified mesophase $\left(M_{x}\right)$ showing a marble texture.

35 As can be seen from this table, the phenoxybenzoic acid derivatives 3a $\sim \mathbf{c}$ are not liquid-crystalline but crystalline. On the other hand, the derivative $\mathbf{3 d}$ is only liquid-crystalline. All the compounds $\mathbf{3}$ are so sublime at high temperatures especially after the clearing point that no samples were in the DSC cell and gave 40 no DSC peaks on the $1^{\text {st }}$ cooling and the $2^{\text {nd }}$ heating runs (Table 2). Since the derivative $\mathbf{3 d}$ is so sublime at high temperatures, it was very difficult to observe the liquid-crystalline texture by a polarizing microscope (POM). When this derivative was gradually heating from rt, it completely sublimated and ${ }_{45}$ disappeared before reaching the mesophase temperature range.
Therefore, the sample was inserted into the hot stage preheated at a certain temperature under the clearing point (= cp) of $246{ }^{\circ} \mathrm{C}$. The set temperature was lowered little by little from the cp and observed by the POM step by step. At last we could observe the 50 liquid-crystalline texture at $220{ }^{\circ} \mathrm{C}$ as shown a photomicrograph in Fig. 4. As can be seen from this photomicrograph, the crystalline phase $\mathrm{K}$ was changing into a marble texture with fluidity characteristic to a nematic phase ${ }^{19}$. . However, it rapidly sublimed within one minute so that the mesophase could not be 55 identified unambiguously. Hence, this mesophase was denoted as an unidentified mesophase $\left(M_{x}\right)$. Nevertheless, this means that we can also obtain calamitic liquid crystals substituted by bulky groups instead of long alkyl chains. The present phenoxybenzoic acid derivative $\mathbf{3 d}$ is the first example of flying-seed-like 60 calamitic liquid crystal, so far as we know.

As can be seen from Table 2, the enthalpy of $167 \mathrm{~kJ} / \mathrm{mol}$ between $220^{*}$ and $246{ }^{\circ} \mathrm{C}$ of $\mathbf{3 d}$ seems to be too big even for the combined enthalpies of melting $\left(K-M_{x}\right)$ and clearing $\left(M_{x}-I . L.\right)$ in comparison with general mesogenic compounds. Although it is 65 not clear at the present time, the sublimation enthalpy might be

Table 2 Phase transition sequences for the flying-seed-like calamitic nPh-PhOBA (3a d), and desolventized Mitsubishi-Mark-shaped (nPhPhOBA $)_{3}$ Tb (4a d) liquid crystals

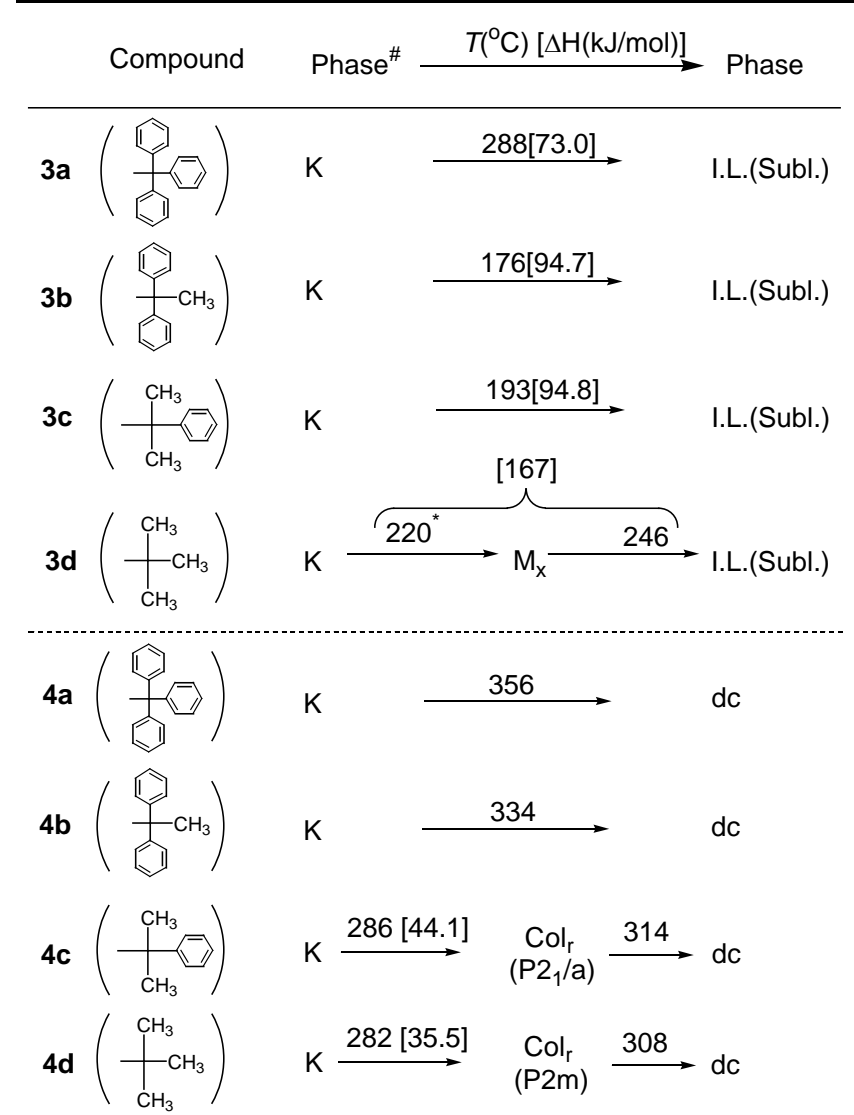

${ }^{\#}$ Phase nomenclature: $\mathrm{K}=$ crystal, $\mathrm{M}_{\mathrm{x}}=$ unidentified mesophase, $\mathrm{Col}_{\mathrm{r}}=$ 70 rectangular columnar mesophase, I.L.(Subl.) = isotropic liquid (accompanied by vigorous sublimation) and $\mathrm{dc}=$ decomposition .

All the phase transition temperatures with enthalpy changes were determined with DSC for the $1^{\text {st }}$ heating run, except for the asteriskmarked temperature which was observed only with POM. The 75 decomposition temperatures were determined with TGA as the onset temperatures. 
included. The compound 3d gave the most vigorous sublimation nature among the compounds 3a-d. Hence, it would give the biggest enthalpy among them.

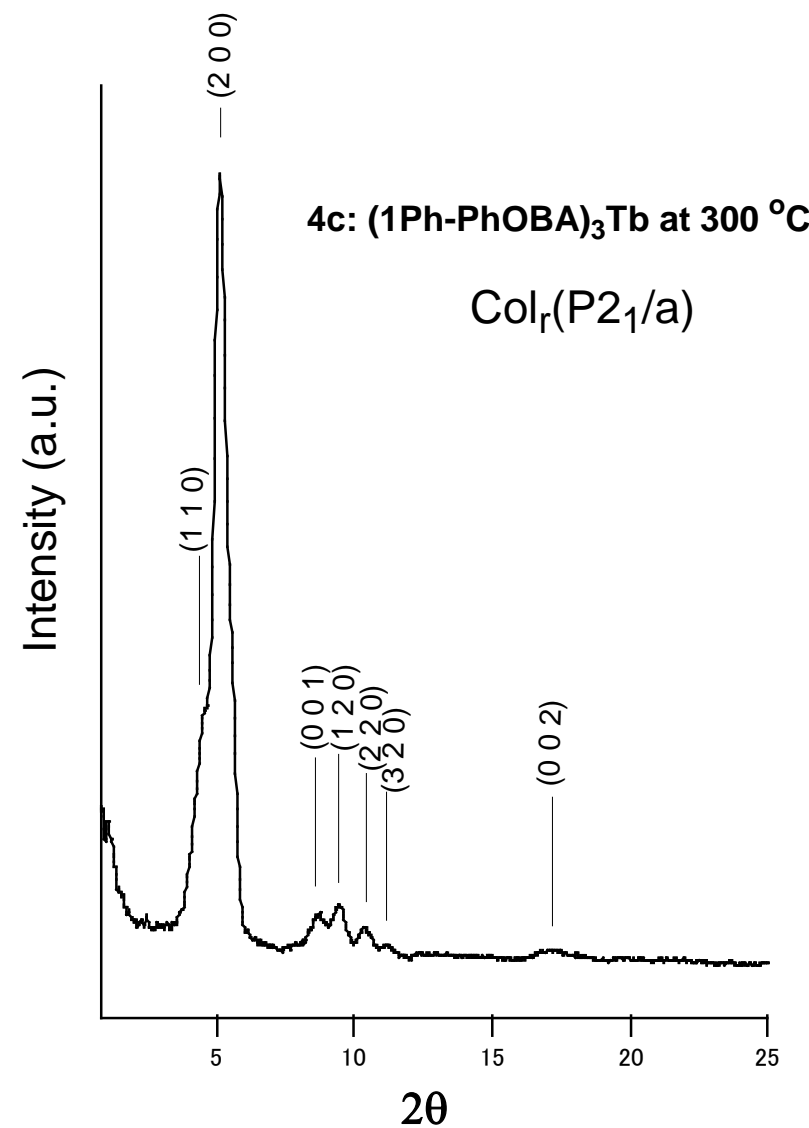

As mentioned above, the bulky phenyl-group-substituted 5 phenoxybenzoic acid derivatives $3 \mathbf{a} \sim \mathbf{c}$ are not liquid-crystalline. It may be attributable to too small core in comparison with the

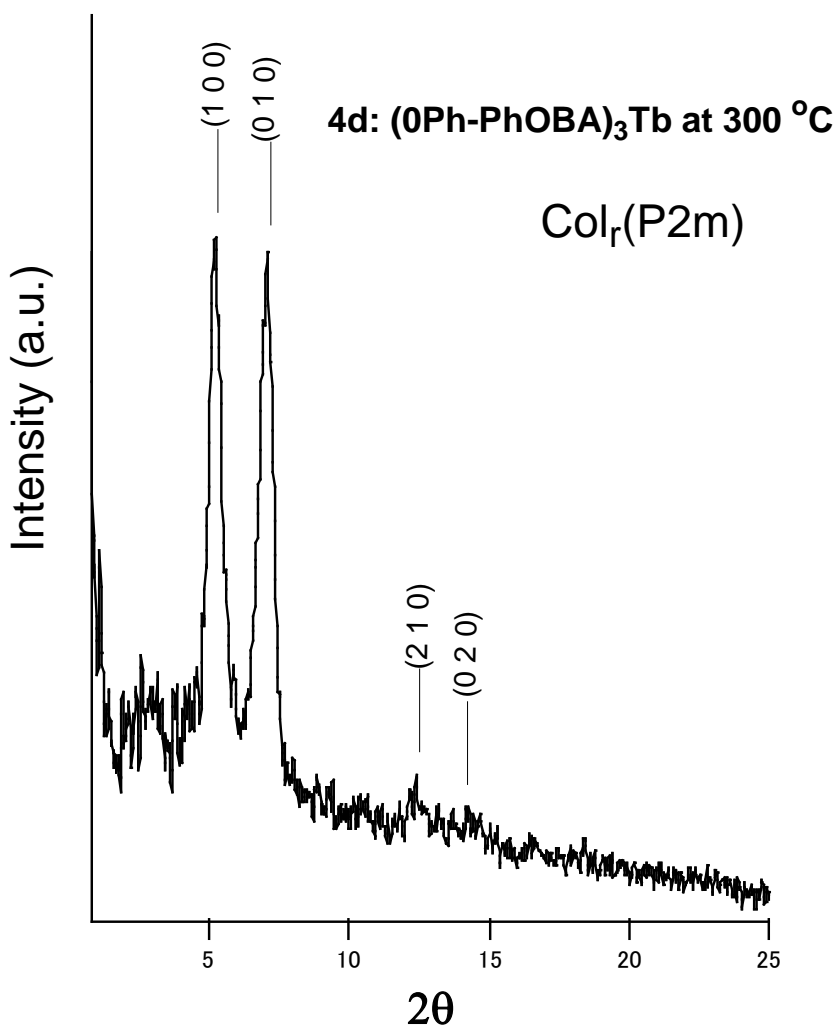

Fig. 5 X-ray diffraction patterns of (1Ph-PhOBA) $)_{3} \mathbf{T b}(\mathbf{4 c})$ and (0Ph-PhOBA) $)_{3} \mathbf{T b}(\mathbf{4 d})$ at $300^{\circ} \mathrm{C}$.

10 Table 3 X-ray data of (1Ph-PhOBA) $)_{3}$ Tb (4c) and (0Ph-PhOBA) $)_{3}$ Tb (4d).

\begin{tabular}{|c|c|c|c|}
\hline \multirow{2}{*}{$\begin{array}{l}\text { Compound } \\
\text { Mesophase }\end{array}$} & \multicolumn{2}{|c|}{ Spacing $/ \AA$} & Miller indices \\
\hline & Observed & Calculated & $(h k l)$ \\
\hline \multicolumn{4}{|c|}{ 4c: (1Ph-PhOBA)з Tb } \\
\hline Colr $(\mathrm{P} 21 / \mathrm{a})$ at $300^{\circ} \mathrm{C}$ & 19.6 & 19.6 & $\left(\begin{array}{lll}2 & 0 & 0\end{array}\right)$ \\
\hline$a=39.2 \AA$ & 17.3 & 17.3 & $\left(\begin{array}{lll}1 & 1 & 0\end{array}\right)$ \\
\hline$b=19.3 \AA$ & 10.1 & 10.1 & $\left(\begin{array}{llll}0 & 0 & 1\end{array}\right)^{\# \#}$ \\
\hline$c=10.1 \AA$ & 9.37 & 9.36 & (1 20 ) \\
\hline \multirow[t]{3}{*}{$Z=4.0$ for $\rho=1.00^{*}$} & 8.53 & 8.64 & $\left(\begin{array}{lll}2 & 2 & 0\end{array}\right)$ \\
\hline & 7.87 & 7.75 & (3 2 0) \\
\hline & 5.14 & 5.07 & $\left(\begin{array}{llll}0 & 0 & 2\end{array}\right)^{\#}$ \\
\hline \multicolumn{4}{|l|}{ 4d: (OPh-PhOBA)з Tb } \\
\hline $\mathrm{Col}(\mathrm{P} 2 \mathrm{~m})$ at $300^{\circ} \mathrm{C}$ & 16.9 & 16.9 & $\left(\begin{array}{lll}1 & 0 & 0\end{array}\right)$ \\
\hline$a=16.9 \AA$ & 12.5 & 12.5 & $\left(\begin{array}{lll}0 & 1 & 0\end{array}\right)$ \\
\hline \multirow[t]{2}{*}{$b=12.5 \AA$} & 7.14 & 6.99 & $\left(\begin{array}{lll}2 & 1 & 0\end{array}\right)$ \\
\hline & 6.20 & 6.23 & $\left(\begin{array}{lll}0 & 2 & 0\end{array}\right)$ \\
\hline
\end{tabular}

\#\# =stacking distance between dimers. \# =stacking distance between monomers. $*=$ assumed value.

bulkier substituents. Thus, these flying-seed-like compounds
15 suggest that the balance of core size and bulkiness of substients is very important to obtain mesomorphism.

Mesomorphism of (nPh-PhOBA) ${ }_{3}$ Tb(III) $(n=1,0: 4 c, d)$

As can be seen from Table 2, the Mitsubishi-Markshaped terbium complexes $\mathbf{4 a}$ and $\mathbf{4 b}$ are not liquid-crystalline 20 but crystalline until their very high decomposition temperatures. On the other hand, $\mathbf{4 c}$ and $\mathbf{4 d}$ exhibit a mesophase at very high temperatures. It is very interesting that the mesomorphism of the phenoxybenzoic aids derivatives $\mathbf{n P h}-\mathbf{P h O B A}(\mathrm{n}=0 \sim 3)$ was nduced only by the bulky substituent $\mathbf{d}(\mathrm{n}=0)$, but that the 25 mesomorphism of their corresponding terbium complexes (nPhPhOBA) ${ }_{3}$ Tb(III) (n=0 3) was induced by the bulky substituents c and $\mathbf{d}(\mathrm{n}=1,0)$. On the other hand, the mesomorphism of the $\mathrm{PcCu}$ complexes (nPh-PhOBA) $)_{\mathbf{4}} \mathbf{P c C u}(\mathrm{n}=0 \sim 3)$ in our previous work was induced by all the bulky substituents $\mathbf{a}, \mathbf{b}, \mathbf{c}$ and $\mathbf{d}$ $30(n=0 \sim 3)^{12)}$. Therefore, balance of core size and bulkiness of substients is very important to obtain mesomorphism for flyingseed-like compounds.

Fig. 5 shows the $\mathrm{X}$-ray diffraction patterns of $\mathbf{4 c}$ and $\mathbf{4 d}$ at $300{ }^{\circ} \mathrm{C}$. These corresponding X-ray data are listed in Table 3. As 35 can be seen from this table, the Mitsubishi-Mark-shaped terbium complexes 4c and 4d give rectangular columnar mesophases, $\mathrm{Col}_{\mathrm{r}}\left(\mathrm{P} 2_{1} / \mathrm{a}\right)$ and $\mathrm{Col}_{\mathrm{r}}(\mathrm{P} 2 \mathrm{~m})$, respectively. The small symmetrical difference between them may be originated from the difference of 
bulkiness of the substituted groups of $\mathbf{c}$ and $\mathbf{d}$. Interestingly, (1Ph-PhOBA $)_{3} \mathrm{~Tb}(\mathbf{4 c})$ showed two stacking distances of dimers and monomers at 10.1 and $5.14 \AA$, respectively. This means that an equilibrium between dimers and monomers exists in the 5 columns. The Mitsubishi-Mark-shaped terbium complexes 4c may form anti-parallel dimers in which one Mitsubishi-Mark stacks on another Mitsubishi-Mark rotated by 60 degree.

As mentioned above, the present terbium complexes $\mathbf{4 c}$ and 4d exhibit a mesophase at very high temperatures, which is 10 characteristic to flying-seed-like liquid crystals ${ }^{12)}$.

\section{3-3. Temperature-dependent fluorescence spectra}

Temperature-dependent fluorescence spectra of the thin films of $\mathbf{4 c}$ and $\mathbf{4 d}$ are shown in Fig.6. In Fig. 7 are plotted the logarithm of the intensities at $545 \mathrm{~nm}$ versus temperature. As can 15 be seen from these figures, the intensities decrease with
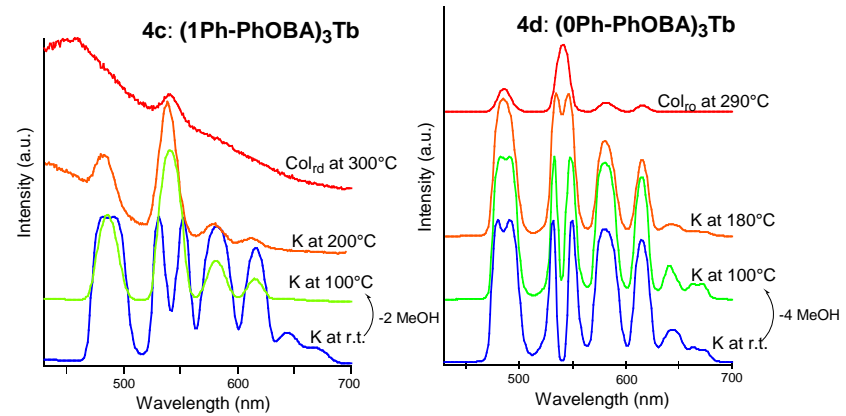

Fig.6 Temperature-dependent fluorescence spectra of (1PhPhOBA) $)_{3} \mathrm{~Tb} \cdot 2 \mathrm{MeOH}(4 \mathrm{c})$ and $(0 \mathrm{Ph}-\mathrm{PhOBA})_{3} \mathrm{~Tb} \cdot 4 \mathrm{MeOH}(4 \mathrm{~d})$.

increasing temperature ${ }^{20 \sim 22)}$. Big deformation of the spectrum of 20 4c at $300{ }^{\circ} \mathrm{C}$ corresponds to considerable decomposition of the sample in the air for the long measurement time. The decomposition temperature $314{ }^{\circ} \mathrm{C}$ listed in Table 2 was the measured value with TGA under nitrogen atmosphere. The present temperature-dependent fluorescence spectral 25 measurements were carried out in the air for long time. Therefore, the decomposition in the air started even at temperatures lower than the TGA decomposition temperatures, and progressed for long time. As the matter of fact, the colour of the sample measured at $300{ }^{\circ} \mathrm{C}$ changed from white to brown.

30 Although there have been several reports that the fluorescence intensities change with phase transitions ${ }^{2023}$, the present terbium complexes did not show discernible drastic changes for their desolventizing and phase transitions, as can be seen from Figure 7. Therefore, the fluorescence spectral changes of the present 35 terbium complexes only depend on their thermal stability.

\section{Conclusion}

We have synthesized novel flying-seed-like calamitc phenoxybenzoic acid derivatives, nPh-PhOBA (3a $\sim$ ), and their Mitsubishi-Mark-shaped terbium complexes, (nPh-PhOBA) $\mathbf{T} \mathbf{T b} \cdot$ $40 \mathbf{~ m M e O H ~ ( 4 a \sim d ) , ~ a n d ~ i n v e s t i g a t e d ~ t h e i r ~ m e s o m o r p h i s m . ~}$

The phenoxybenzoic acid derivatives $\mathbf{3 a} \sim \mathbf{c}$ are not liquidcrystalline but crystalline, whereas the derivative $\mathbf{3 d}$ is only liquid-crystalline. The derivative $\mathbf{3 d}$ shows an unidentified mesophase $\left(M_{x}\right)$ at very high temperatures from $220{ }^{\circ} \mathrm{C}$ to $246{ }^{\circ} \mathrm{C}$. $45 \mathrm{It}$ is the first example of flying-seed-like calamitic liquid crystal,

\section{4c: (1Ph-PhOBA) ${ }_{3} \mathrm{~Tb} \cdot 2 \mathrm{MeOH}$}

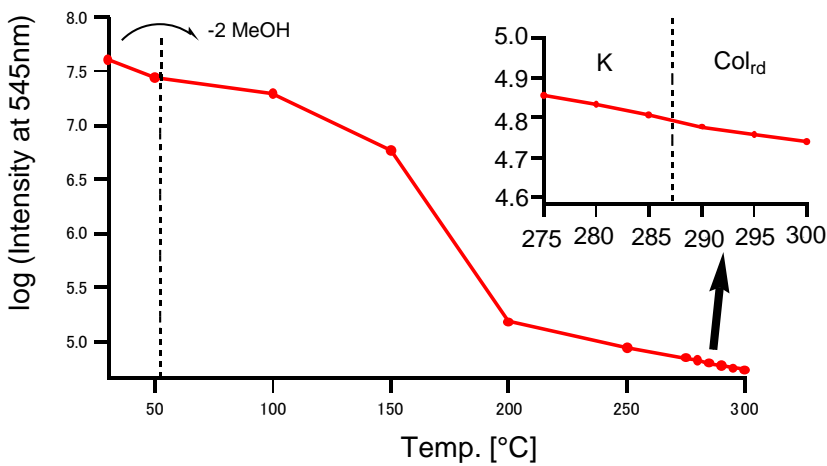

4d: (OPh-PhOBA) ${ }_{3} \mathrm{~Tb} \cdot 4 \mathrm{MeOH}$

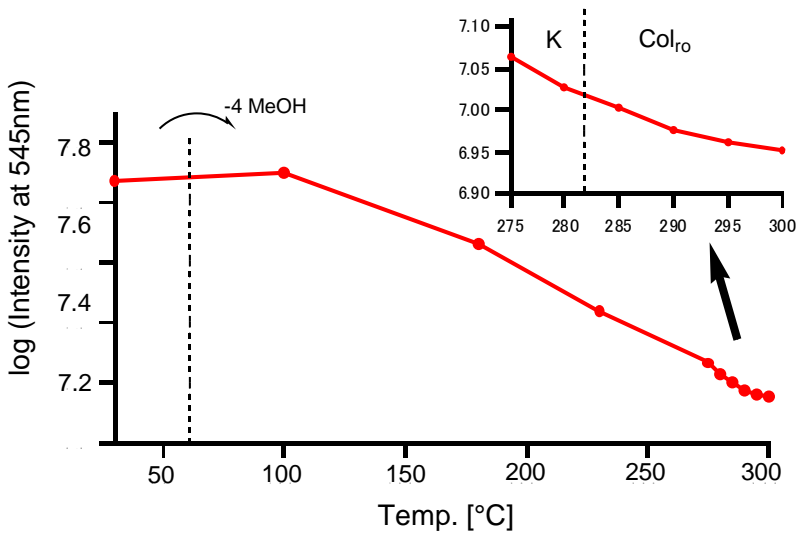

Fig.7 Fluorescent intensities at $545 \mathrm{~nm}$ versus temperature for (1PhPhOBA) $)_{3} \mathrm{~Tb} \cdot 2 \mathrm{MeOH}(4 \mathrm{c})$ and $(0 \mathrm{Ph}-\mathrm{PhOBA})_{3} \mathrm{~Tb} \cdot 4 \mathrm{MeOH}(4 \mathrm{~d})$.

so far as we know. On the other hand, the terbium complexes $4 \mathbf{a}$ 50 and $\mathbf{4 b}$ are not liquid-crystalline but crystalline, whereas $\mathbf{4 c}$ and 4d exhibit rectangular columnar mesophases $\operatorname{Col}_{\mathrm{r}}\left(\mathrm{P} 2_{1} / \mathrm{a}\right)$ and $\mathrm{Col}_{\mathrm{r}}(\mathrm{P} 2 \mathrm{~m})$, respectively. It is very interesting that the $\mathrm{PcCu}$ complexes (nPh-PhOBA) ${ }_{4} \mathbf{P c C u}$ in our previous work are liquid crystalline for all the bulky substituents $\mathbf{a}, \mathbf{b}, \mathbf{c}$ and $\mathbf{d}$.

55 Thus, it becomes apparent that both calamitic and columnar mesomorphism can be induced by substitution of bulky groups instead of long alkyl chains, and that balance of core size and bulkiness of substients in flying-seed-like compounds is very important to obtain mesomorphism. We believe that this is a new ${ }_{60}$ guideline for induction of mesomorphism by using bulky groups instead of long alkyl chains.

\section{Notes}

${ }^{a}$ Smart Material Science and Technology, Interdisciplinary Graduate School of Science and Technology, Shinshu University, 1-15-1 Tokida,

65 Ueda, 386-8567, Japan. Tel\&FAX: +81-268-21-5492; E-mail:

ko52517@shinshu-u.ac.jp

${ }^{b}$ Department of General Education, Nagano National College of Technology, Nagano, 381-8550, Japan. E-mail: itaya@ge.naganonct.ac.jp

$70 \dagger$ Part 2: Ref. 12 in this paper.

\section{References}

1. D. Vorländer, Ber. Dtsch. Chem. Ges., 1911, 43, 3120-3125 
2. D. Demus, H. Sackmann and K. Seibert, Wiss. Z. Univ. Halle, Math.Nat. R. 1970, 19, 47-62.

3. P. Ferloni, M. Sanesi, P. L. Tonelli and P. Franzosini, Z. Naturforsch., 1978, 33, 240-242.

5 4. M. Sanesi, P. Ferloni, G. Spinolo and P. L. Tonelli, Z. Naturforsch., 1978, 33, 386-388.

5. R. van Deun, J. Ramaekers, P. Nockemann, K. van Hecke, L. van Meervelt and K. Binnemans, Eur. J. Inorg. Chem., 2005, 563-571.

6. K. Ohta, T. Shibuya and M. Ando, J. Mater. Chem., 2006, 16, 36353639.

7. S. Basurto, S. Garcia, A. G. Neo, T. Torroba, C. F. Marcos, D. Miguel, J. Barbera, M. B. Ros and M. R. de la Fuente, Chem. Eur. J., 2005, 11, 5362-5376.

8. M. Shimizu, M. Nata, K. Watanabe, T. Hiyama and S. Ujiie, Mol. Cryst. Liq. Cryst., 2005, 441, 237-241.

9. M. Shimizu, M. Nata, K. Mochida, T. Hiyama, S. Ujiie, M. Yoshio and T. Kato, Angew. Chem. Int. Ed., 2007, 46, 3055-3058.

10. N. Usol'tseva, V. Bykova, G. Ananjeva, N. Zharnikova and E. Kudrik, Mol. Cryst. Liq. Cryst. 2004, 411, 1371-1378.

20 11. N. Zharnikova, N. Usol'tseva, E. Kudrik and M. Theakkat, J. Mater. Chem., 2009, 19, 3161-3167.

12. Y. Takagi, K. Ohta, S. Shimosugi, T. Fujii and E. Itoh, J. Mater. Chem., 2012, 22, 14418-14425.

13. Mitsubish-Mark: see http://www.mitsubishi.com/e/group/mark.html.

25 14. R. A. Evangelista, H. E. Wong, E. F. G. Templeton, T. Granger, B. Allore and A. Pollak, Anal. Biochem., 1992, 203, 218-226.

15. T. Shibuya, Appendix 1 in Master Thesis, Shinshu University, Ueda, 2006.

16. N. K. Lokanath, D. Krishne Gowda, D. Revannasiddaiah, M. M. M.

$30 \quad$ Abdoh, M. A. Sridhar and J. Shashidhara Prasad, Mol. Cryst. Liq. Cryst., 1998, 317, 153-164.

17. A. Sato, Y. Yanamura, K. Saito and M. Sorai, Liq. Cryst., 1999, 26, 1185-1195.

18. T. Shimogaki, S. Dei, K. Ohta and A. Matsumoto, J. Mater. Chem., 2011, 21, 10730-10737.

19. D. M. Small, M. C. Bourges and D. G. Dervichian, Biochim. Biophys. Acta, 1966, 125, 563-580.

20. E. Venuti, R. G. Della Valle, I. Bilotti, A. Brillante, M. Cavallini, A. Cal ò and Y. H. Geerts, J. Phys. Chem. C, 2011, 115, 12150-12157.

40 21. C.-T. Liao, H.-H. Chen, H.-F. Hsu, A. Poloek, H.-H. Yeh, Y. Chi, K.W. Wang, C.-H. Lai, G.-H. Lee, C.-W. Shih, and P.-T. Chou, Chem, Eur, J., 2011, 17, 546-556.

22. C. V. Yelamaggad, A. S. Achalkumar, D. S. S. Rao and S. K. Prasad, J. Org. Chem., 2009, 74, 3168-3171.

45 23. R. Cristian, J. Eccher, I. H. Bechtold, C. N. Tironi, A. A. Vieira, F. Molin and H. Gallardo, Langmuir, 2012, 28, 11590-11598. 